\title{
Relación Entre La Duplicidad De La Marca Y La Penetración De La Marca En Productos De Consumo Habitual
}

\author{
Dra. Laura Fischer \\ Facultad de Contaduría y Administración, \\ Universidad Nacional Autónoma de México \\ Mtro. Jorge Espejo \\ Universidad Autónoma del Estado de Morelos, México
}

doi: 10.19044/esj.2016.v12n16p412 URL:http://dx.doi.org/10.19044/esj.2016.v12n16p412

\begin{abstract}
In order to examine the association between a brand and its penetration for a series of everyday consumption products, a quantitative study was conducted on 60 households in Mexico City. During a 12 week time period the recurrent purchase behavior of participants was recorded and analyzed. Results revealed that a high percentage of participants who purchased a product identified with a specific Brand, also tended to purchase other products of the same Brand.
\end{abstract}

Keywords: Brand duplicity, customary purchasing, Brand penetration

\section{Resumen}

Con el propósito de examinar la posible relación entre la marca y su penetración, en productos de compra para consumo habitual, se realizó un estudio cuantitativo mediante la técnica de panel en 60 hogares de la ciudad de México. Durante un periodo de 12 semanas se registraron y analizaron los comportamientos de compra recurrente de esos hogares. Los resultados muestran que un alto porcentaje de personas que compra un producto identificado por una marca especifica también tienden a comprar diferentes productos de la misma marca.

Palabras clave: duplicidad de marca, compra habitual, penetración de la marca

\section{Introducción}

Para los fabricantes de productos y servicios de consumo final, el comportamiento de compra del consumidor es una tema que está en 
incesante cambio por lo que requiere de un estudio continuo, (Fischer, 2011); al principio, el consumo se limitaba a las ventas (desplazamiento de unidades físicas y monetarias); posteriormente, la experiencia y los datos recogidos del mercado permitieron descubrir que algunos consumidores repiten la compra de la marca o producto con más frecuencia que otros y que no necesariamente satisfacen todas sus necesidades a través de esa marca, por lo que tienden a comprar otras marcas. (Kotler, 2001).

Brown desde 1953 describe como la repetición de compra de cualquier producto/marca de consumo recurrente tiende a seguir un patrón común, lo que permite establecer una teoría con base empírica y discernir sencillas normas de interpretación para evaluar cualquier observación particular, por ejemplo, si la incidencia de repetición de los compradores mes a mes, es alta o baja, o, es simplemente normal; Ehrenberg por su parte considera que ese comportamiento sistemático de compra puede ser reconocido como lealtad del consumidor hacia la marca. (Ehrenberg, 1972, 1988, 1990, 2004).

\section{Marco teórico}

En el entorno competitivo actual es insuficiente monitorear de manera aislada la cuota de mercado, Perles, Ramón \& Sevilla (2014) son de la opinión de que es estratégicamente conveniente acompañar esta métrica con otras medidas de desempeño que la complementen, sus hallazgos, determinan que una de estas medidas es la "frecuencia media de compra", por lo tanto, este binomio de indicadores resulta muy atractivo pues permite al mercadólogo decidir, eventualmente, si su estrategia de crecimiento consistirá en ganar cuota de mercado arrebatando clientes a la competencia o aprovechando la tendencia creciente del mercado.

Brown (1952, 1953), Cunningham (1956) y Ehrenberg (1970) aseguran que los compradores no satisfacen necesariamente todas sus necesidades a través de una marca, por lo que también tienden a comprar otras marcas; esta conclusión es compartida y confirmada en investigaciones realizadas por Jeuland (1979), Baldinger (1997), Wright \& Riebe (2010)y Rogers, Morgan \& Foxall (2015).

Por su parte Chrysochou, Krystallis \& Giraud (2012), Fader, Hardie $\&$ Sen (2014) y Sheth (1991)sugieren desde una perspectiva comportamental de la lealtad de marca, que cuando alguien compra una marca que es buena, según los estímulos de su propia experiencia o por influencia de grupos de referencia, aumenta la probabilidad de volverla a comprar, esto significa para Jeuland (1979) que la probabilidad de recompra de una marca aumenta según su frecuencia de compra y su capacidad de ofrecer resultados satisfactorios; por su parte Ehrenberg $(1972,1988)$ describe los patrones predecibles en el 
comportamiento de repetición de compra que se ha demostrado aplican en cientos de categorías de productos, países y el tiempo.

En un estudio llevado a cabo por Fischer \& Espejo 2016 ${ }^{12}$, confirmaron tres generalizaciones propuestas por Brown (1952, 1953), Cunningham (1956), Ehrenberg y Goodhardt (1970), Fader y Schmittlein (1993), y Bhattacharya (1997): a) Repetición de compra: la conducta repetida de compra, de cualquier producto de consumo recurrente, tiende a seguir un patrón común de orden cero; b) duplicidad de la marca: los compradores no satisfacen necesariamente todas sus necesidades a través de una marca, por lo que tienden a comprar también otras marcas y c) el fenómeno Doble Riesgo (Double Jeopardy): la participación de mercado y la frecuencia media de compra tiende a mantener valores paralelos tanto en las marcas grandes como en las pequeñas.

Para académicos, investigadores y practicantes de la mercadotecnia estos hallazgos resultan trascendentes dada la importancia de las decisiones que están implícitas en temas estratégicos como segmentación, posicionamiento, diferenciación, innovación, benchmarking, tolerancia competitiva, atracción, retención y lealtad del consumidor, entre otras.

Los resultados de esa investigación también reportan que el coeficiente de duplicidad de marcas es un indicador de gran ayuda para el mercadólogo al permitirle identificar con precisión las características y preferencias comparativas de los segmentos del mercado que satisfacen sus necesidades y expectativas con las diversas marcas disponibles en una misma categoría de producto, planteando consecuentemente opciones para resolver dilemas de posicionamiento; Lees \& Wright (2009) llegaron a un resultado similar en un estudio sobre la industria de la radio, descubrieron que el mercado está particionado (segmentado) y que la selección que hacen los radioescuchas no es entre diferentes marcas de radio, como se cree popularmente, más bien es entre los formatos o categorías de producto: estaciones de palabra y estaciones de música.

Esa investigación descubría planteamientos estratégicos adicionales derivados del monitoreo al coeficiente de duplicidad de marcas, uno es el benchmarking ${ }^{13}$, estar consciente de los motivos que guían al mercado a consumir las marcas de la competencia conjuntamente con la propia, permite

\footnotetext{
${ }^{12} \mathrm{El}$ análisis de este resultado se encuentra en dos artículos titulados: "Comportamientos de Lealtad a la Marca en Hogares de la Ciudad de México" (Fischer, L., Espejo, J., 2016a). y Comportamiento de duplicidad de marca, sustitución de marca, lealtad compartida entre marcas y doble riesgo en compras de productos de consumo habitual en hogares de la Ciudad de México" (Fischer, L., Espejo, J, 2016b) que se encuentran en próxima publicación

${ }^{13}$ El benchmarking es una herramienta estratégica, y en la mercadotecnia está destinada a lograr propuestas competitivas eficientes en la oferta de marcas y productos, Fischer y Espejo (2011).
} 
adoptar las mejores prácticas a las circunstancias y características de la empresa, así como diseñar una estrategia de diferenciación sostenible, pero que sobre todo sea rentable dado que no resulta extraño darse cuenta que se desperdician los recursos invertidos en la diferenciación de las marcas gestionadas por encontrarse estas dentro del esquema de duplicidad de marca del mercado meta; Romaniuk, Sharp \& Ehrenberg (2007) descubrieron, que la diferenciación juega un papel más limitado en la competencia entre marcas, su estudio también reportó que existe bajo nivel en la percepción de diferenciación entre marcas de competencia directa, sin embargo y a pesar de esta falta de diferenciación percibida, en ambas investigaciones se observa que los clientes siguen repitiendo la compra de dos o más marcas de la misma categoría de producto.

Existen otros estudios relacionados con el coeficiente de duplicidad de marcas y la lealtad compartida, como señala la teoría de la competencia multi-mercados planteada por Jayachandran, Gimeno \& Varadarajan (1999), aquí una de las implicaciones más importantes para la estrategia de mercadotecnia es el impacto esperado en la tolerancia competitiva, todo mercadólogo debe tomar conciencia de que las acciones estratégicas implantadas para sus marcas también generan respuestas favorables en las marcas competidoras dada la interdependencia que los consumidores tienen con dos o más marcas dentro de la misma categoría de producto.

Ehrenberg y Goodhard ", (Ehrenberg A. G.1970:78) definen la Ley de la duplicidad de la marca como: "para cualquier par de marcas de un producto de compra frecuente, y en un periodo dado, la proporción de compradores de la marca A que también compra la marca B generalmente es directamente proporcional al número de compradores de la marca $\mathrm{B}$, esos consumidores de dos marcas (consumidores duplicantes) compran cada marca con una frecuencia promedio que tiende a ser una fracción fija de la frecuencia con la cual los compradores de cada marca hacen sus compras".

\section{Método}

Con el objetivo de encontrar la relación entre la duplicidad de la marca y la penetración de la marca en productos de compra de consumo habitual. Se realizó un estudio de naturaleza longitudinal/transversalcuantitativa a través de panel de hogares, donde en una hoja Excel por hogar durante un periodo de 12 semanas, se registraron, analizaron y midieron los comportamientos de compra recurrente de hogares mexicanos radicados en la ciudad de México.

Hipótesis: existe, en general, una relación directa entre la penetración de una marca y la duplicidad de la marca; es decir, las grandes marcas suelen compartir en promedio más clientes entre si y menos con las pequeñas marcas. 
La investigación utilizó un universo de trabajo de 60 hogares de los alumnos de un grupo de la Facultad de Contaduría y Administración de la UNAM, se observó que todos los hogares eran residentes de la zona sur del área metropolitana de la Ciudad de México (ver El estudio fue llevado a cabo durante 12 semanas en los meses de septiembre a noviembre del 2015; los alumnos entregaban un ticket de compra y registraban en hoja Excel los productos de consumo recurrente adquiridos en el hogar.

Tabla 1. Hogares participantes de la Investigación. Elaboración propia

\begin{tabular}{|c|c|c|c|c|}
\hline Hogares & AB & $\mathbf{C +}$ & $\mathbf{C}$ & C- \\
\hline Participantes & 4 & 16 & 13 & 27 \\
\hline Proporción & $6.3 \%$ & $26.3 \%$ & $20.9 \%$ & $45.0 \%$ \\
\hline AMAI ${ }^{14}$ & & & & \\
Nacional & $7.6 \%$ & $13.7 \%$ & $17.0 \%$ & $17.1 \%$ \\
\hline Hogares DF & 186,430 & 336,065 & 417,015 & 419,468 \\
\hline
\end{tabular}

Para el análisis de la información; se seleccionaron cinco categorías consideradas representativas de los hábitos alimenticios y de higiene de los hogares del Distrito Federal tomadas de las categorías alimentos, higiene, belleza y uso doméstico pertenecientes al canasto de productos Nielsen (Canasto Nielsen, 2014). : Cereal, yogurt, papel higiénico, shampoo para cabello y atún enlatado; los datos de la categoría cereal fueron tabulados por marca de fabricante, para el resto de las categorías los datos se tabularon por marca de producto.

\section{Hallazgos}

Los resultados obtenidos en las cinco categorías de productos, se presentan por separado en donde hemos realizado un gráfico para cada grupo de producto; en ellos, se muestra la relación entre la duplicidad de la marca y la penetración de ella en los hogares.

A cada grupo se obtuvo el coeficiente de correlación para determinar la existencia de duplicidad e intercambio existente en los consumidores.

14 Asociación Mexicana de Agencias de Investigación de Mercado y Opinión Pública (AMAI, 2015) 
Grafico 1 Relación entre duplicidad de marca y penetración de marca Cereal
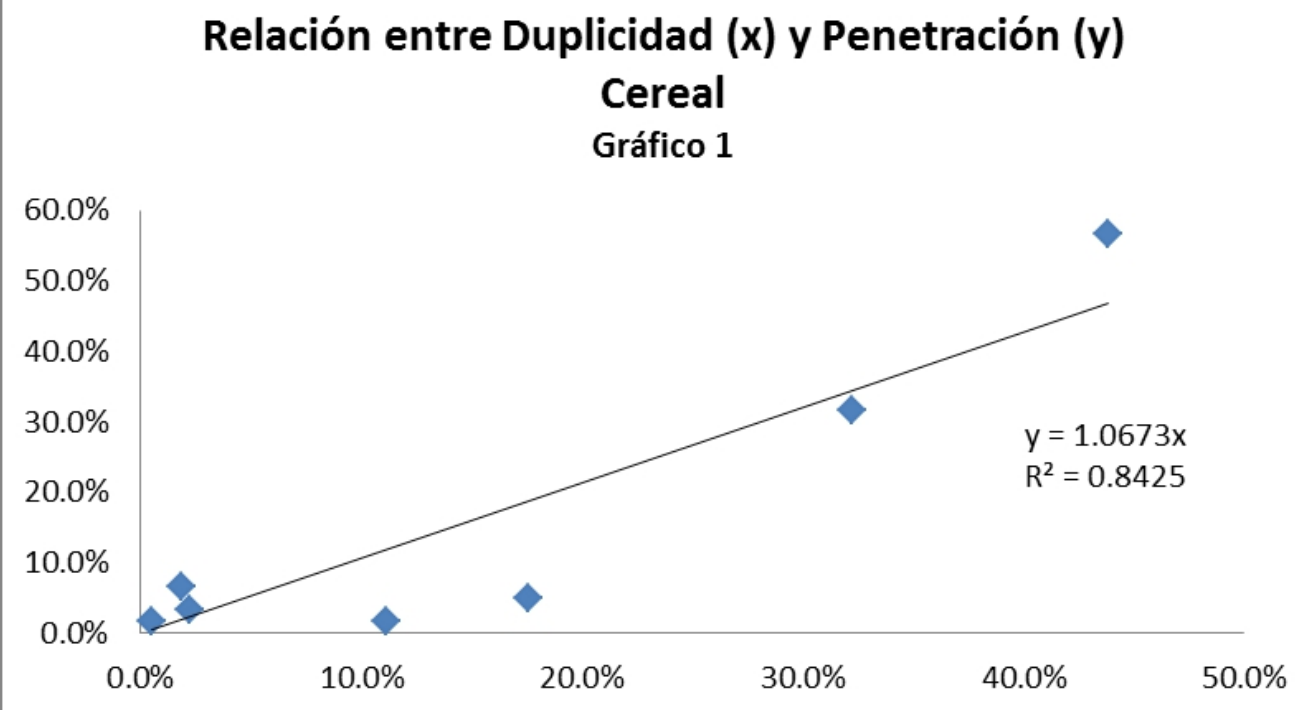

En el gráfico 1, cada punto es una marca de cereal participante en el estudio, el gráfico muestra para cada marca la relación que existe entre su duplicidad y su penetración, podemos observar para cada una de las marcas que los niveles promedio de duplicación de compra declinan de derecha a izquierda (eje de las " $x$ ") conforme la penetración declina también de arriba hacia abajo (eje de las "y"), este es el resultado esperado de la ley de la duplicación de la compra de marcas, en otras palabras, las marcas competidoras suelen compartir en promedio más clientes con las grandes marcas y menos con las pequeñas marcas.

El valor 1.0673 es el coeficiente de duplicación en la compra de marcas (o coeficiente de intercambio), este valor expresa la relación esperada entre el tamaño de una marca A y la proporción media de los usuarios de otras marcas que se esperaría compraran también la marca $\mathrm{A}$ en un período de tiempo determinado, además, sirve para calcular la duplicación esperada para cada una de las marcas.

$\mathrm{R}^{2}$ se refiere al coeficiente de determinación, y el valor .8425 expresa la proporción de variación conjunta entre las variables: Duplicidad y Penetración, se considera que este valor muestra una relación entre las variables muy alta. 
Grafico 2 Relación entre duplicidad de marca y penetración de marca de Yogurt

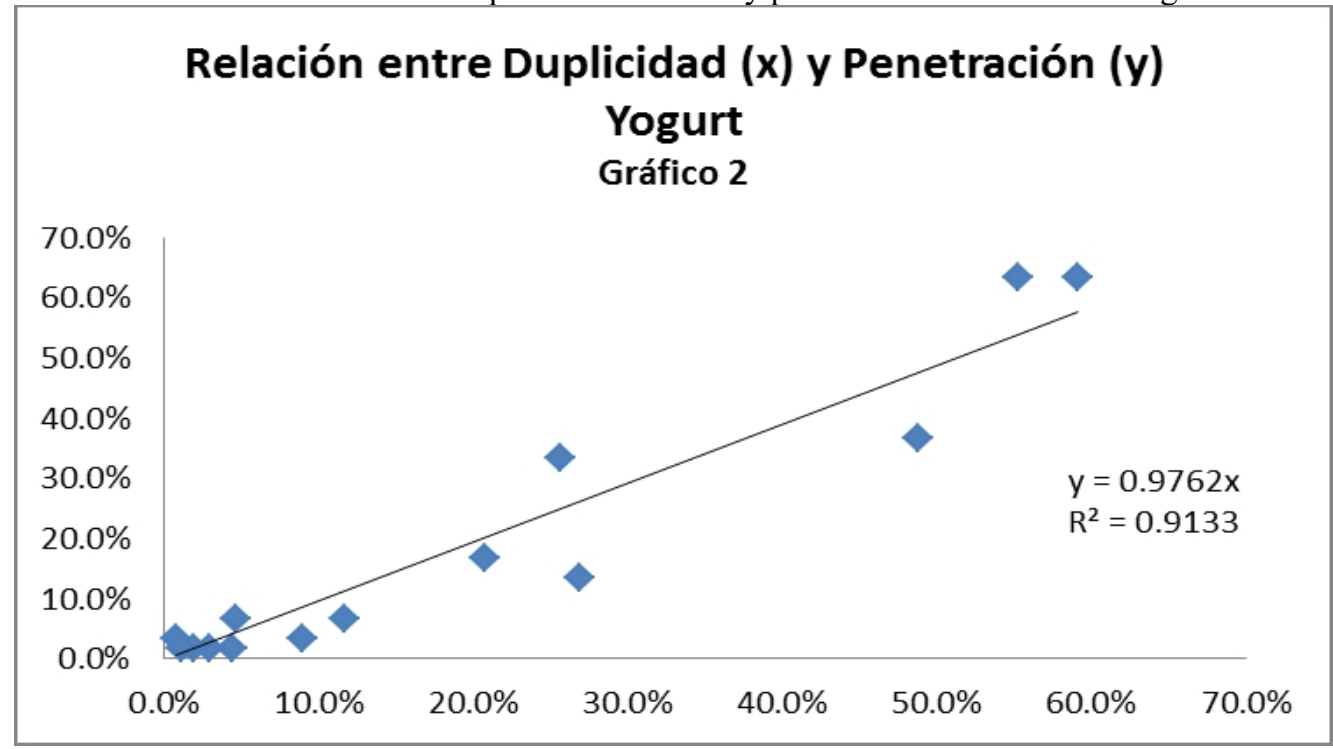

En el gráfico 2 cada punto se refiere a una marca de yogurt participante en el estudio, el gráfico muestra para cada marca la relación que existe entre su duplicidad y su penetración, podemos observar para cada una de las marcas que los niveles promedio de duplicación de compra declinan de derecha a izquierda (eje de las " $x$ ") conforme la penetración declina también de arriba hacia abajo (eje de las "y"), este es el resultado esperado de la ley de la duplicación de la compra de marcas, en otras palabras, las marcas competidoras suelen compartir en promedio más clientes con las grandes marcas y menos con las pequeñas marcas.

El valor .9762 es el coeficiente de duplicación en la compra de marcas (o coeficiente de intercambio), este valor expresa la relación esperada entre el tamaño de una marca A y la proporción media de los usuarios de otras marcas que se esperaría compraran también la marca $\mathrm{A}$ en un período de tiempo determinado, además, sirve para calcular la duplicación esperada para cada una de las marcas.

$\mathrm{R}^{2}$ se refiere al coeficiente de determinación, y el valor .9133 expresa la proporción de variación conjunta entre las variables: Duplicidad y Penetración, se considera que este valor muestra una relación entre las variables muy alta. 
Grafico 3 Relación entre duplicidad de marca y penetración de marca de papel higiénico.

\section{Relación entre Duplicación (x) y Penetración Papel Higiénico Gráfico 3}

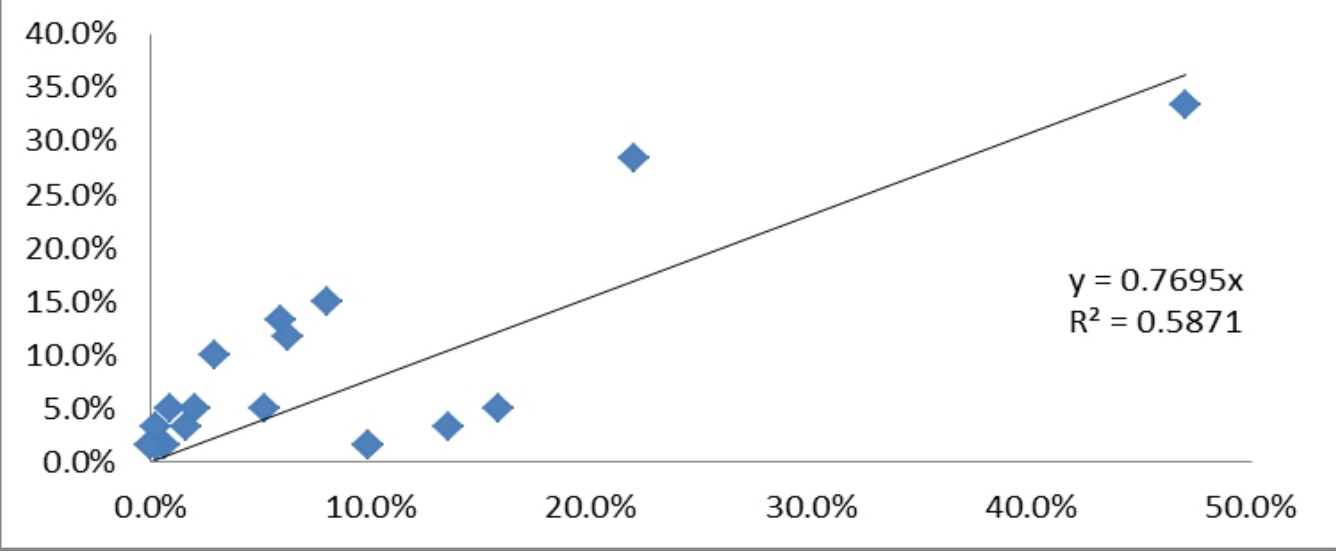

En el gráfico 3 se observa cada punto como una marca de papel higiénico participante en el estudio, el gráfico muestra para cada marca la relación que existe entre su duplicidad y su penetración, podemos observar para cada una de las marcas que los niveles promedio de duplicación de compra declinan de derecha a izquierda (eje de las "x") conforme la penetración declina también de arriba hacia abajo (eje de las "y"), este es el resultado esperado de la ley de la duplicación de la compra de marcas, en otras palabras, las marcas competidoras suelen compartir en promedio más clientes con las grandes marcas y menos con las pequeñas marcas.

El valor .7695 es el coeficiente de duplicación en la compra de marcas (o coeficiente de intercambio), este valor expresa la relación esperada entre el tamaño de una marca A y la proporción media de los usuarios de otras marcas que se esperaría compraran también la marca A en un período de tiempo determinado, además, sirve para calcular la duplicación esperada para cada una de las marcas.

$\mathrm{R}^{2}$ se refiere al coeficiente de determinación, y el valor .5871 expresa la proporción de variación conjunta entre las variables Duplicidad y Penetración, se considera que este valor muestra una relación entre las variables moderada. 
Grafico 4 Relación entre duplicidad de marca y penetración de marca de shampoo.

\section{Relación entre Duplicación (x) y Penetración (y) Shampoo \\ Gráfico 4}

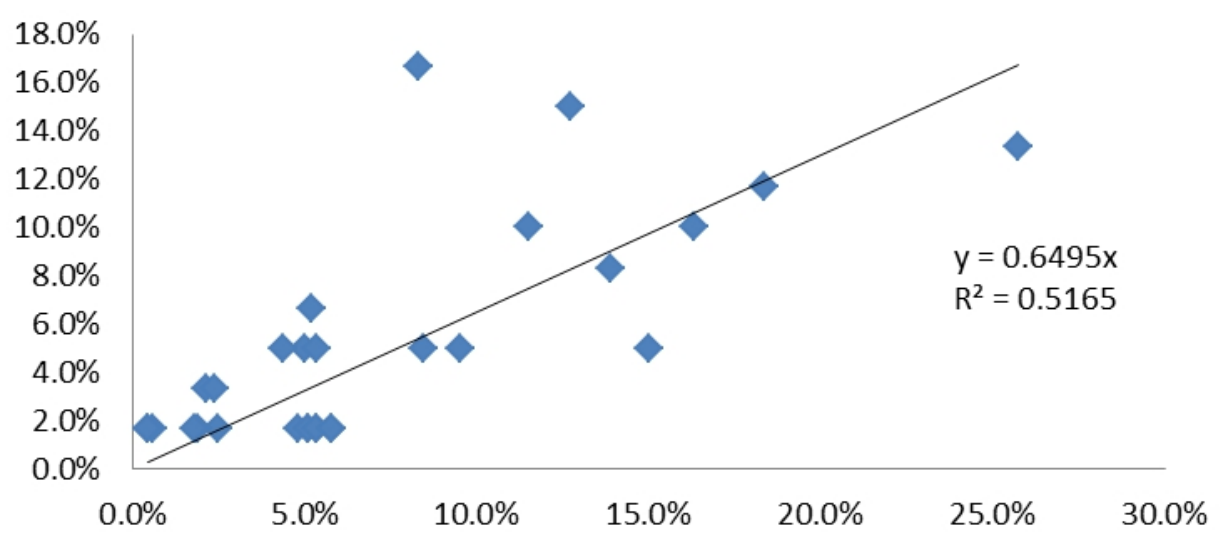

En el gráfico 4, muestra cada punto es de una marca de shampoo participante en el estudio, el gráfico muestra para cada marca la relación que existe entre su duplicidad y su penetración, podemos observar para cada una de las marcas que los niveles promedio de duplicación de compra declinan de derecha a izquierda (eje de las "x") conforme la penetración declina también de arriba hacia abajo (eje de las "y"), este es el resultado esperado de la ley de la duplicación de la compra de marcas, en otras palabras, las marcas competidoras suelen compartir en promedio más clientes con las grandes marcas y menos con las pequeñas marcas.

El valor .636 es el coeficiente de duplicación en la compra de marcas (o coeficiente de intercambio), este valor expresa la relación esperada entre el tamaño de una marca A y la proporción media de los usuarios de otras marcas que se esperaría compraran también la marca $\mathrm{A}$ en un período de tiempo determinado, además, sirve para calcular la duplicación esperada para cada una de las marcas.

$\mathrm{R}^{2}$ se refiere al coeficiente de determinación, y el valor .4287 expresa la proporción de variación conjunta entre las variables Duplicidad y Penetración, se considera que este valor muestra una relación entre las variables moderada.

Particularmente en este gráfico podemos observar que un par de marcas aparecen más alejadas de la línea de tendencia, estas desviaciones sugieren la existencia en el mercado de algunas 'particiones' o agrupación de marcas que compiten más intensamente entre sí. 


\section{Conclusion}

Este estudio nos ha permitido concluir que las diversas marcas que adquieren los compradores duplicantes comparten características como (a) insumos intrínsecos, (b) razones de uso y (c) venta en puntos de distribución del mismo vecindario.

Los hallazgos son de gran relevancia pues al mercadólogo le permiten comprender mejor cómo es que "operan" los mercados en términos de productos y marcas cuya compra para los consumidores es de naturaleza duplicante a lo largo del tiempo.

Confirmamos que en general, la proporción de compradores de una marca que también compra una segunda marca es principalmente una función de esta segunda marca.

Finalmente, también observamos que los mercados tienden a repartirse o dividirse en grupos de marcas y que los compradores suelen compartir sus compras entre marcas a las que atribuyen la misma importancia, en otras palabras, las marcas grandes comparten más clientes con las marcas grandes y menos con las marcas pequeñas, lo mismo sucede entre marcas pequeñas; definimos aquí a las marcas grandes en función a su penetración en el mercado.

\section{References:}

AMAI. (7 de 12 de 2015). Asociación Mexicana de Agencias de Investigación de Mercado y Opinión Pública. Obtenido de AMAI: http://nse.amai.org/nseamai2/

Baldinger, A. R. (1997). The Jeopardy in Double Jeopardy. Journal of Advertising Research 37, 6, 37-49.

Bhattacharya, C. (1997). Is Your Brand's Loyalty too much, too little, or just right?: Explaining Deviations in Loyalty from the Dirichlet Norm. International Journal of Research in Marketing, 14, 421-435.

Brown, G. (1952, 1953). Brand-Loyalty - Fact or Fiction? New York: Advertising Age \# 24 January.

Enero, 75-6.

(1953). Brand-Loyalty - Fact or Fiction? Advertising Age, \# 24,

Cannon, T. E. (1970). Regularities in sole buying. British Journal of Marketing, 4 (2), 80-6.

Chrysochou, P. K. (2012). Quality assurance labels as drivers of customer loyalty in the case of traditional food products. Food quality and preference, 25(2), 156-62.

Cunningham, R. (1956). Brand Loyalty - What, Where, How Much? Harvard Business Review, 34, 116-28.

Ehrenberg. (1970). A Note on Never Buyers. Journal of Marketing Research, 7 Nov, 536-38. 
(1972, 1988). Repeat Buying. Nueva York: Charles Griffin and Company, Londres y Oxford University press.

(1970). A Model of Multi-Brand Buying. Journal of Marketing Research, Vol. VII, 77-84.

July, 82-91.

(1990). Double Jeopardy Revisited. Journal of Marketing, 54

(2004). Understanding Brand Performance Measures: Using Dirichlet Benchmarks. Journal of Business Research, 57, 1307-25.

Fader, P. H. (2014). Stochastic models of buyer behavior. The History of Marketing Science, 17, 165.

Fader, P. S. (1993). Excess Behavioral Loyalty for High-Share Brands: Deviation from the Dirichlet Model for Repeat Purchasing: Deviations from the Dirichlet Model for Repeat Purchase. Journal of Marketing Research, 30 (No. 4), 478-93.

Fischer, L. E. (2011). Mercadotecnia. México: McGraw-Hill.

INEGI. (2011). Panorama Socioeconómico del Distrito Federal. México: Instituto Nacional de Estadística y Geografía.

Jacoby, J. C. (1978). Brand Loyalty: Measurement and Management. John Wiley \& Sons, New York, 509-11.

Jayachandran, S. G. (1999). The Theory of Multimarket Competition: A Synthesis and Implications for Marketing Strategy. Journal of Marketing Vol. 63 (July), 49-66.

Jeuland, A. (1979). Brand Choice Inertia as One Aspect of the Notion of Brand Loyalty, , p. Management Science, Vol. 25, $n^{\circ} .7,671-81$.

Kotler, P. (2001). Dirección de Marketing La edición del milenio $10^{a}$ Edición, 718 pp. México: Pearson Prentice Hall.

Kuehn, A. (1962). Consumer Brand Choice, a learning process ? Journal of advertising research 2, December, 10-17.

Lees, G. W. (2009). Does the Duplication of Purchase Law Apply to Radio Listening? Australasian Marketing Journal, 1-7.

Lipstein, B. (1959). The dynamics of brand loyalty and brand switching. Procedings Fifth annual conference, 1-10.

Luce, R. (1959). Individual Choice Behavior: A Theoretical Analysis. New York: John Wiley \& Sons.

Morrison, D. (1969). Conditional Trend Analysis: A Model that Allows for Nonusers. Journal of Marketing Research, Vol VI Ago, 342-6.

Nielsen, C. (24 de 03 de 2016). Nielsen. Obtenido de The Nielsen Company: http://www.nielsen.com/mx/es/search.html?q=canastos\&sp_cs=UTF-8

Pamies, D. (2003). La fidelidad del cliente en el ámbito de los servicios: un análisis de la escala intenciones de comportamiento. Investigaciones Europeas de Dirección y Economía de la Empresa, 9(2), 189-204. 
Perles, J. R. (2014). La cuota de mercado como indicador de competitividad en los destinos turísticos: Sentido y Limitaciones . Cuadernos de Turismo, $n^{o}$ $34,265-85$.

Ríos, P. (2000). La duplicidad de una marca: Caso Nike. Iuris: Actualidad y práctica del derecho, (41), 74+6.

Rogers, A. M. (2015). Triple jeopardy in a behavioral perspective. The Routledge Companion to Consumer Behavior Analysis, 150.

Romaniuk, J. S. (2007). Evidence concerning the importance of perceived brand differentiation. Australasian Marketing Journal 15 (2), 42-54.

Romo, H. (2009). Niveles Socioeconómico AMAI. México: Instituto de Investigaciones Sociales SC.

Sabavala, D. M. (1977). A model of TV show loyalty. Journal of Advertising Research, 17(6), 35-43.

Sharp, B. R. (2005). Does Triple Jeopardy Exist for Retail Chains? Journal of Empirical Generalisations in Marketing, 1-9.

Sheth, J. N. (1991). Why We Buy What We Buy: A Theory of Consumption Values. Journal of Business Research, 22, 159-170.

Subodh, B. a. (1996). An Investigation of Jeopardy Effects in Store Choice. Journal of Retailing and Consumer Services, 3 (No. 3), 129-33.

Uncles, M. a. (1995). Grocery Store Patronage. The International Review of Retail, Distribution \& Consumer Research, 287-302.

Wright, M. R. (2010). Double jeopardy in brand defection. European Journal of Marketing, 44(6), 860-73. 